\title{
Genome survey sequencing provides clues into glucosinolate biosynthesis and flowering pathway evolution in allotetrapolyploid Brassica juncea
}

\author{
Jinghua Yang ${ }^{1,2}$, Ning Song ${ }^{1,2}$, Xuan Zhao ${ }^{1,2}$, Xiaohua Qi ${ }^{3}$, Zhongyuan $\mathrm{Hu}^{1,2}$ and Mingfang Zhang ${ }^{1,2^{*}}$
}

\begin{abstract}
Background: Brassica juncea is an economically important vegetable crop in China, oil crop in India, condiment crop in Europe and selected for canola quality recently in Canada and Australia. B. juncea $(2 n=36, A A B B)$ is an allotetraploid derived from interspecific hybridization between B. rapa $(2 n=20, A A)$ and B. nigra $(2 n=16, B B)$, followed by spontaneous chromosome doubling.
\end{abstract}

Results: Comparative genome analysis by genome survey sequence (GSS) of allopolyploid B. juncea with B. rapa was carried out based on high-throughput sequencing approaches. Over 28.35 Gb of GSS data were used for comparative analysis of $B$. juncea and B. rapa, producing $45.93 \%$ reads mapping to the $B$. rapa genome with a high ratio of single-end reads. Mapping data suggested more structure variation (SV) in the B. juncea genome than in $B$. rapa. We detected 2,921,310 single nucleotide polymorphisms (SNPs) with high heterozygosity and 113,368 SVs, including 1-3 bp Indels, between B. juncea and B. rapa. Non-synonymous polymorphisms in glucosinolate biosynthesis genes may account for differences in glucosinolate biosynthesis and glucosinolate components between $B$. juncea and B. rapa. Furthermore, we identified distinctive vernalization-dependent and photoperioddependent flowering pathways coexisting in allopolyploid B. juncea, suggesting contribution of these pathways to adaptation for survival during polyploidization.

Conclusions: Taken together, we proposed that polyploidization has allowed for accelerated evolution of the glucosinolate biosynthesis and flowering pathways in B. juncea that likely permit the phenotypic variation observed in the crop.

Keywords: Brassica juncea, Comparative genome analysis, Flowering pathway, Genome survey sequencing, Glucosinolate biosynthesis

\section{Background}

The Brassicaceae family includes approximately 3,700 species in 350 genera with diverse characteristics, many of which are of agronomic importance as vegetables, condiments, fodder and oil crops [1]. The genus Brassica contains the majority of crop species of Brassicaceae family. Of particular importance are the cole crop and vegetable species B. rapa, B. oleracea, B. napus, and B. juncea as sources of oils and vegetables. Because of their agricultural

\footnotetext{
* Correspondence: mfzhang@zju.edu.cn

'Laboratory of Germplasm Innovation and Molecular Breeding, Institute of Vegetable Science, Zhejiang University, Hangzhou 310058, P. R. China ${ }^{2}$ Key laboratory of Horticultural Plant Growth, Development \& Quality Improvement, Ministry of Agriculture, Hangzhou 310058, P. R. China Full list of author information is available at the end of the article
}

importance, genome components of several Brassica species have been characterized in detail over the past few years [2-4]. The genomes of three diploid species, $B$. rapa $(\mathrm{AA}, 2 \mathrm{n}=20), B$. nigra $(\mathrm{BB}, 2 \mathrm{n}=16)$, and B. oleracea $(\mathrm{CC}$, $2 \mathrm{n}=18$ ), have been shown to contain triplicate homologous counterparts of corresponding segments in the Arabidopsis genome due to whole-genome triplication that occurred approximately 12-17 million years ago [1,5]. Additional natural allopolyploidization events in the last 10,000 years, have resulted in the creation of three allotetraploid hybrids, B. juncea (AABB, $2 \mathrm{n}=36$ ), B. napus (AACC, $2 \mathrm{n}=38$ ) and B. carinata $(\mathrm{BBCC}, 2 \mathrm{n}=34)$ [6-10]. $B$. juncea is used as a vegetable in China and Southeastern Asia, and is a source of oil in India and Europe. The species possesses unique traits that include much wider

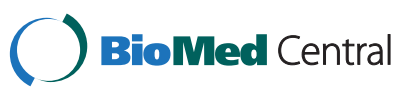


morphological variation in leafy types, root type, stem type, seed stalk type and oil type [11]. B. juncea has been reported to contain higher glucosinolates than other Brassica species [12]. Glucosinolates are of higher value to human nutrition that may reduce the risk of cancer incidence. In addition, they are toxic to some soil-borne plant pathogens, hence, accounting for their selection $[13,14]$.

The recent accomplishment of genome sequencing and annotation of $B$. rapa [5], combined with the available genome sequence data for model Arabidopsis in Brassicaceae [15], provide improved strategies for comparative genome analysis and breeding. Attempts to develop a unified comparative genomics system in the Brassicaceae have revealed 24 conserved genomic blocks [4], an extension to the 21 syntenic blocks identified in B. napus [16]. Comparative mapping studies between members of Brassica and Arabidopsis thaliana [16-22], and Arabidopsis thaliana and Capsella rubella [23], together with the identification of an ancestral karyotype (AK) [24], have stimulated interest in the evolutionary processes underlying diversification in the Brassicaceae. Since the allotetraploid species possess much larger genomes than their diploid counterparts in Brassica [2], we expect that novel gene/pathway interactions have emerged in the allotetraploid Brassica species through sub-functionalization and/ or neo-functionalization of paralogs $[25,26]$.

Low coverage genome survey sequences (GSS) can provide information about gene content, polymorphism, functional elements, repetitive elements and molecular markers [27-31]. In some studies, most of the coding sequence in a genome can be surveyed with less than 2 genome coverage [32]. It was possible to recover $38 \%$ of the coding fraction of the mouse-human alignment with only $0.66 \times$ coverage of the pig genome [33] With only $0.1 \times$ coverage, it was possible to generate a considerable amount of biologically useful information and genomic resources for Megaselia scalaris, including identification of repetitive elements, the mitochondrial genome, microsatellites and identification of gene homologs [34]. These studies make a compelling case for low density sequencing in the genomic studies of nonmodel species.

Here, we employed high-throughput sequencing for comparative genome analysis of $B$. juncea and B. rapa to identify genome changes associated with polyploidization that might account for the phenotypic diversity of B. juncea. We showed clues of glucosinolate biosynthesis and flowering pathway evolution occurred in Brassica juncea, likely accounting for some of the phenotypic diversity that is observed. Furthermore, it provides a valuable resource for more focused investigations into the rate and distribution of genomic changes that accompany polyploidization in this species.

\section{Results}

\section{Karyotype of $B$. juncea}

According to the 'U-triangle' theory of Brassicaceae [6], allotetraploid $B$. juncea originated from hybridization of B. rapa (AA, $2 \mathrm{n}=20)$ and $B$. nigra $(\mathrm{BB}, 2 \mathrm{n}=16)$. We identified genomic components of $B$. juncea by genomic in situ hybridization (GISH). The two predicted genomes (A and B) of the allotetraploid were distinguished using genomic DNA from B. rapa and B. nigra as probes representing the putative progenitor genomes. The $20 \mathrm{~A}$ and 16 B chromosomes detected suggest that the two genomes have remained somewhat distinct in B. juncea with no significant genome homogenization and no large-scale translocations between genomes (Figure 1).

\section{Comparative genome analysis of $B$. juncea and $B$. rapa}

After quality evaluation of sequencing data (Additional file 1: Figure S1), a total of $28.35 \mathrm{~Gb}$ high quality data were collected for the $B$. juncea genome and used to compare with whole genome sequence of $B$. rapa. It was feasible to map $45.93 \%$ sequences of the B. juncea GSS data to the genome sequences of $B$. rapa. Of these, only $18.44 \%$ single-end reads were mapped to the genome sequences of $B$. rapa, which indicated more SV in the $B$. juncea genome compared to $B$. rapa. The identity of mapped sequences is $98.14 \%$, which shows a close genetic relationship between $B$. juncea and $B$. rapa (Additional file 1: Table S1). The coverage depth and distribution on chromosomes suggest a high comparison ratio over the $B$. rapa genome (Additional file 1: Figure S2).

Polymorphism analysis identified 2,921,310 SNPs, including $58.53 \%$ transitions, $41.47 \%$ transversions and $58.19 \%$ heterozygosity. We showed the distributions of SNP-type in 10 chromosomes of $B$. rapa genome (Additional file 1: Table S2, Additional file 1: Figure S3). 44,053 SVs were detected as insertions and deletions, with approximately even distributions of SVs across the 10 chromosomes of $B$. rapa genome (Additional file 1: Table $\mathrm{S} 3$, Additional file 1: Figure S3). 69,315 Indel (1-3 bp) polymorphisms were also observed, of which $1 \mathrm{bp}$-sized Indels were most abundant in genome and $3 \mathrm{bp}$-sized Indels were most abundant in coding sequence (Additional file 1: Table S4, Additional file 1: Figure S3). Most SNPs and SVs (including 1-3 bp Indels) were located in exon, intron, transposon, intergenic, TEprotein, TandemRepeat region of genome, others were found in miRNA, tRNA and snRNA coding regions of genome (Table 1). These SNPs cause a relatively high ratio of non-synonymous mutations in genes; for example, 9680 genes were found with $(\geqq 10)$ non-synonymous SNPs. Moreover, 1448 genes coding regions were changed by frame-shift Indels, and we also found 5989 genes have SV within gene coding regions (Table 2). A number of gene functions were found to be 

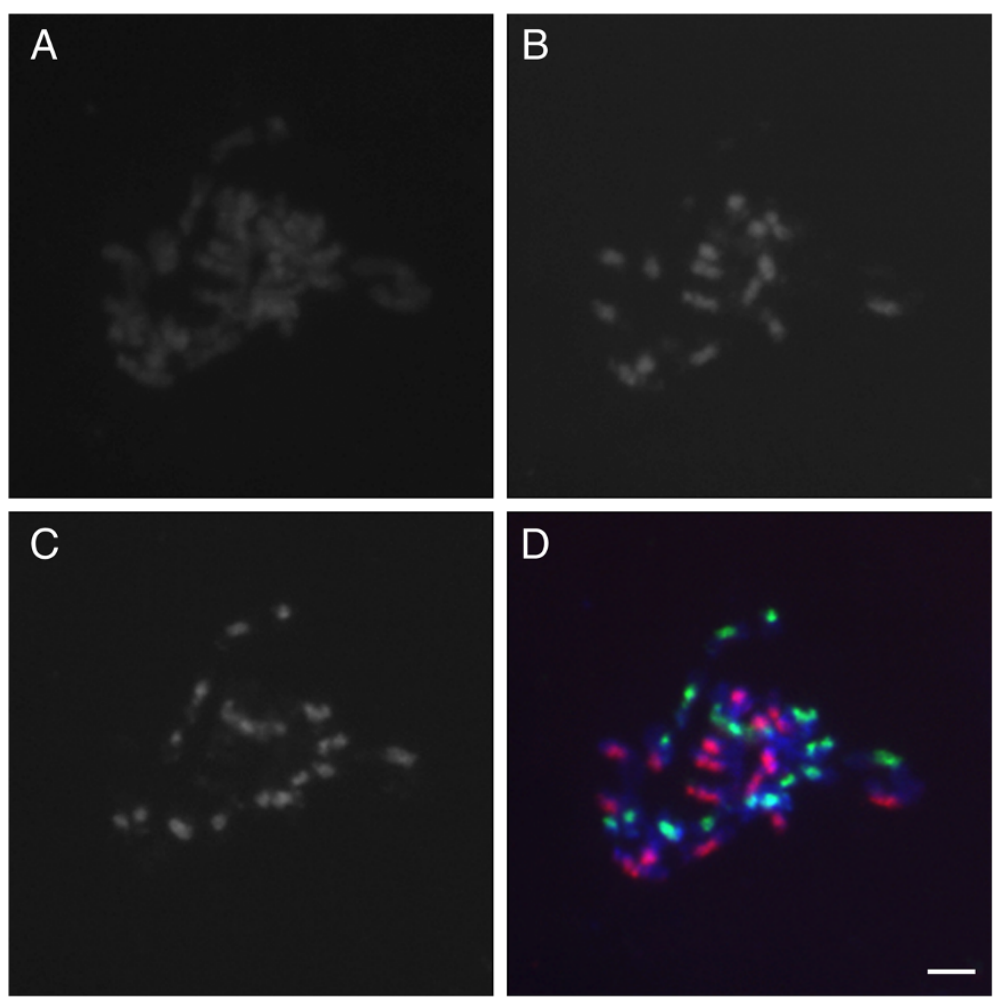

Figure 1 Genomic in situ hybridization analysis of genome component in B. juncea. Metaphase chromosome from root tip cell of $B$. juncea (A), detection of B genome chromosome in B. juncea chromosome (B), detection of A genome chromosome in B. juncea chromosome (C), A and $B$ genomes with red and green fluorescence in B. juncea (D). Bar $=5 \mu \mathrm{m}$.

altered by these mutations based on Non-Redundant $\mathrm{Nu}$ cleotide Database (NT/NR), Cluster of Othologues Groups Proteins Database (COG) and Kyoto Encyclopedia of Genes and Genomes Database (KEGG) database searches (data not shown). Here, we have focused on glucosinolate biosynthesis and flowering pathways in particular.

\section{Glucosinolate biosynthesis genes expression between $B$. juncea and $B$. rapa}

We constructed glucosinolate biosynthesis pathway in $B$. juncea by KEGG analysis. Three biosynthesis pathways were identified from different substrates including methionine, branched-chain amino acid and aromatic amino acid (Figure 2). Among glucosinolate biosynthesis-related genes, we found non-synonymous SNPs and deletion/insertion SV polymorphisms in CYP79F1 (CYP, cytochromes P450), CYP83A1, SUR1 (SUPERROOT1), UGT74B1 (UDP-glucose: thiohydroximate S-glucosyltransferase), SOT16 (sulfotransferase), CYP79A2, CYP83B1, CYP79B2 and CYP79B3 genes (Additional file 1: Table S5), which suggested different genes expressions and glucosinolate components and contents. Gene expression of 6 selected glucosinolate biosynthesis-related genes were investigated in leaves between B. juncea and B. rapa. CYP83A1, CYP79A2 and $C Y P 79 F 1$ expressions were up-regulated in $B$. juncea than $B$. rapa. CYP83B1 expression was down-regulated in $B$. juncea than $B$. rapa. There was no difference in CYP79B2 and SUR1 expressions between B. juncea and $B$. rapa (Figure 3 ). These mutations appear to cause differences in gene expression and glucosinolate content between $B$. juncea and B. rapa.

Table 1 Distribution of SNPs and SVs polymorphisms in genomic components in $B$. juncea

\begin{tabular}{|c|c|c|c|c|c|c|c|c|c|}
\hline Type & Exon & Intron & miRNA & tRNA & snRNA & TEprotein & Transposon & TandemRepeat & Intergenic \\
\hline SNP & 912059 & 276795 & 393 & 789 & 671 & 88942 & 629793 & 59754 & 952114 \\
\hline $\mathrm{SV}^{*}$ & 3769 & 9038 & 14 & 33 & 51 & 2374 & 11901 & 2896 & 13977 \\
\hline Indel* & 3294 & 17253 & 11 & 21 & 19 & 2314 & 15313 & 1459 & 29631 \\
\hline
\end{tabular}


Table 2 Statistics of non-synonymous mutations by SNPs, genes with Frame-shift by Indels and genes with SVs in B. juncea

\begin{tabular}{llll}
\hline $\begin{array}{l}\text { Genes with Non-synonymous } \\
\text { SNPs: total detected SNPs }\end{array}$ & $\begin{array}{l}\text { Genes with(>=10) Non-synonymous } \\
\text { SNPs: total detected SNPs }\end{array}$ & $\begin{array}{l}\text { Genes with Frame-shift Indels: } \\
\text { total detected Indel }\end{array}$ & $\begin{array}{l}\text { Genes with SVs: total } \\
\text { detected SVs }\end{array}$ \\
\hline $35457: 278241$ & $9608: 166533$ & $1448: 1528$ & $5989: 7059$ \\
\hline
\end{tabular}

Glucosinolate component and content between $B$. juncea and $B$. rapa

We checked glucosinolate component and content between B. juncea and B. rapa by HPLC. Of glucosinolate component, sinigrin, gluconapin, glucobrassicanapin, glucobrassicin and 4-Methoxy glucobrassicin were detected in young leaves of B. juncea, of which sinigrin showed very high content with $19.58 \mu \mathrm{mol} / \mathrm{g}$ DW in leaves. Only glucobrassicin, 4-Methoxy glucobrassicin and neoglucobrassicin were detected in young leaves of B. rapa (Figure 4).

The flowering pathway in Brassica juncea

Flowering behavior is an essential feature affecting Brassicaecae crop production. For B. rapa (AA genome), seed vernalization and long-day photoperiod conditions are necessary for flowering (Figure 5-A, B), while only long-day photoperiod conditions promote $B$. nigra flowering, without any need for vernalization treatment (Figure 5-A, B). Interestingly, long-day photoperiod conditions lead to flowering in $B$. juncea regardless of vernalization conditions (Figure 5-C, D). We identified four FLOWERING LOCI C (FLC1, FLC2, FLC3 and FLC5) genes and other flowering pathway-related genes, including CONSTANS (CO), CONSTANS -like (COL), FLOWERING T (FT), LEAFY, SOC1 (SUPPRESSOR OF OVEREXPRESSION OF CO1) and AP1 (APETALA1), in B. juncea. Under vernalization and longday photoperiod conditions, when FLCs gene expression is down-regulated, flowering occurs by an FLC-dependent pathway in B. juncea. Under non-vernalization and long-day photoperiod conditions, flowering occurs by a CONSTANS-dependent pathway, not FLC-dependent, since FLCs genes are still expressed during flowering

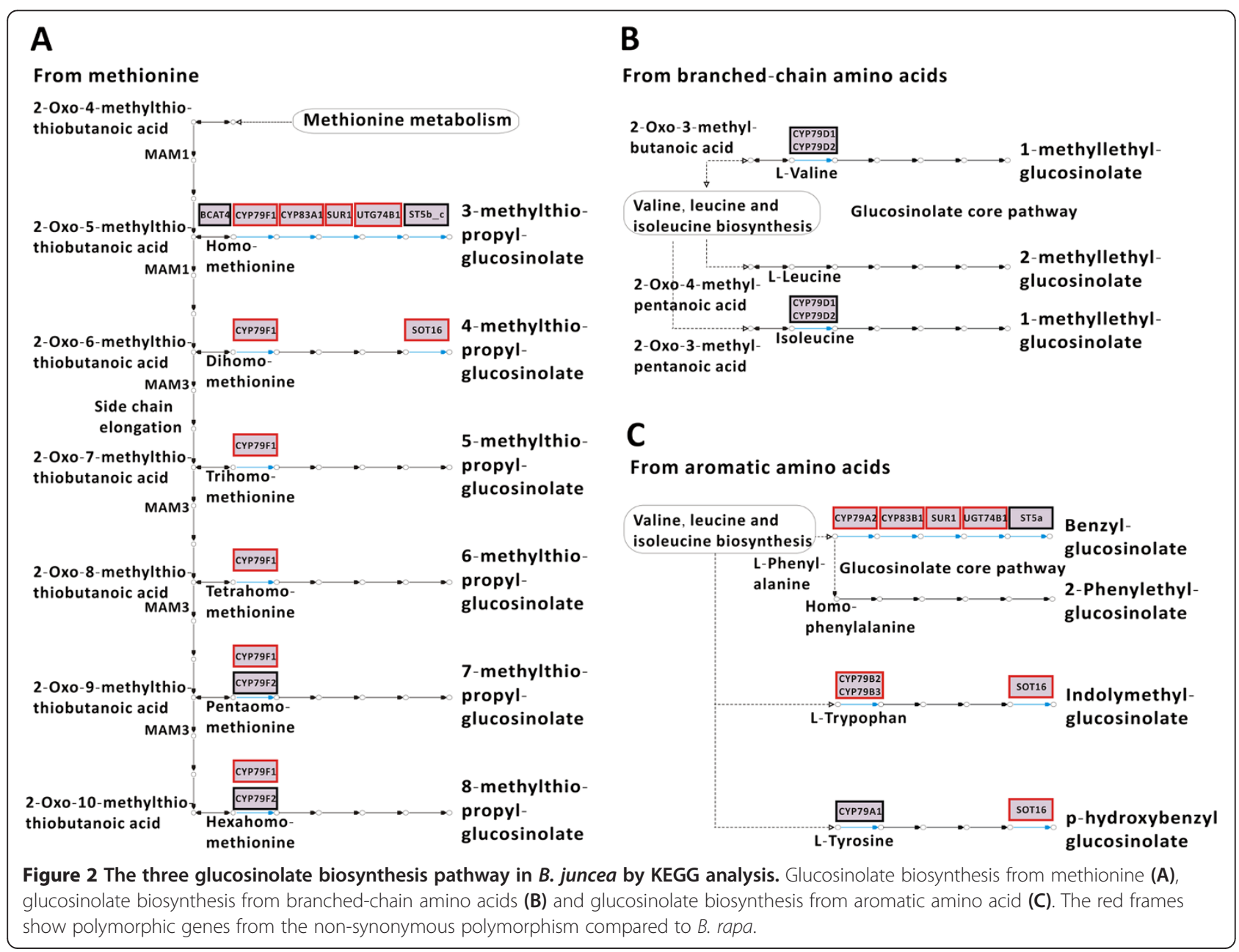



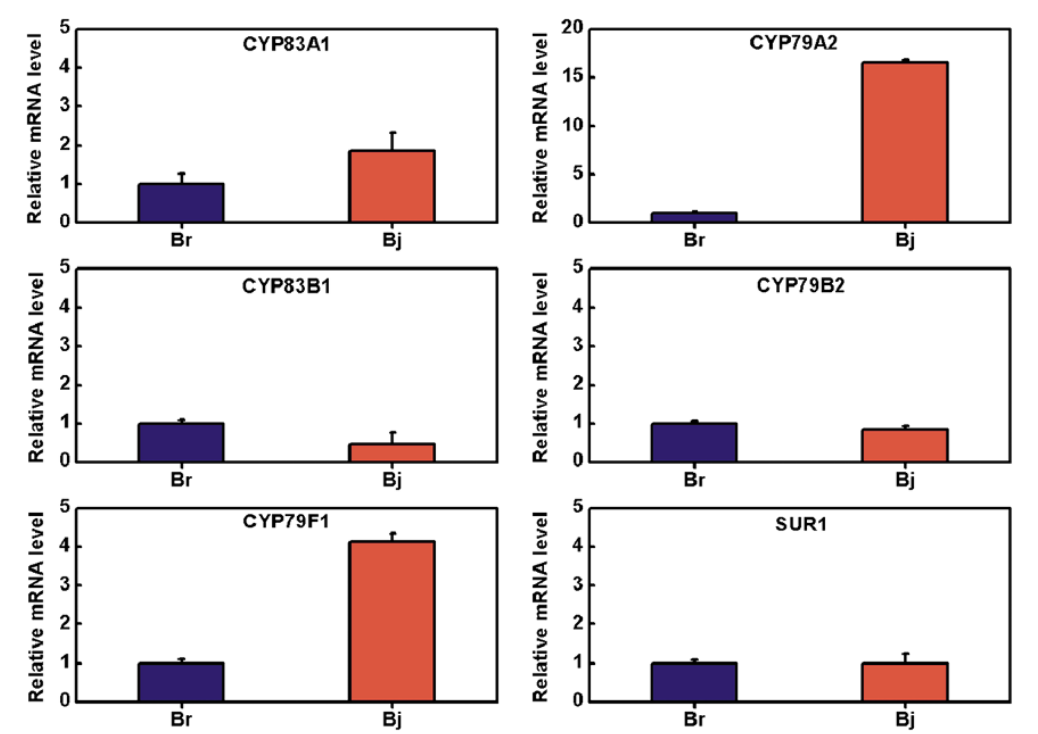

Figure 3 Transcriptional patterns of glucosinolate biosynthesis related genes in B. juncea and B. rapa.

(Figure 5-E). These results indicate that vernalizationand photoperiod-dependent flowering pathways coexist in the allotetraploid B. juncea (Figure 5-F).

\section{Discussions}

Allotetraploid B. juncea possesses unique traits that influence its utility as a vegetable crop in China and oil crop in India; these features emerged after natural hybridization between $B$. rapa and $B$. nigra and allopolyploidization. The A genome (B. rapa) [5] and $C$ genome [35] sequences were recently completed, providing considerable momentum in molecular genetic studies of Brassica. Brassica A/ $\mathrm{B} / \mathrm{C}$ genome phylogeny and evolution is of considerable

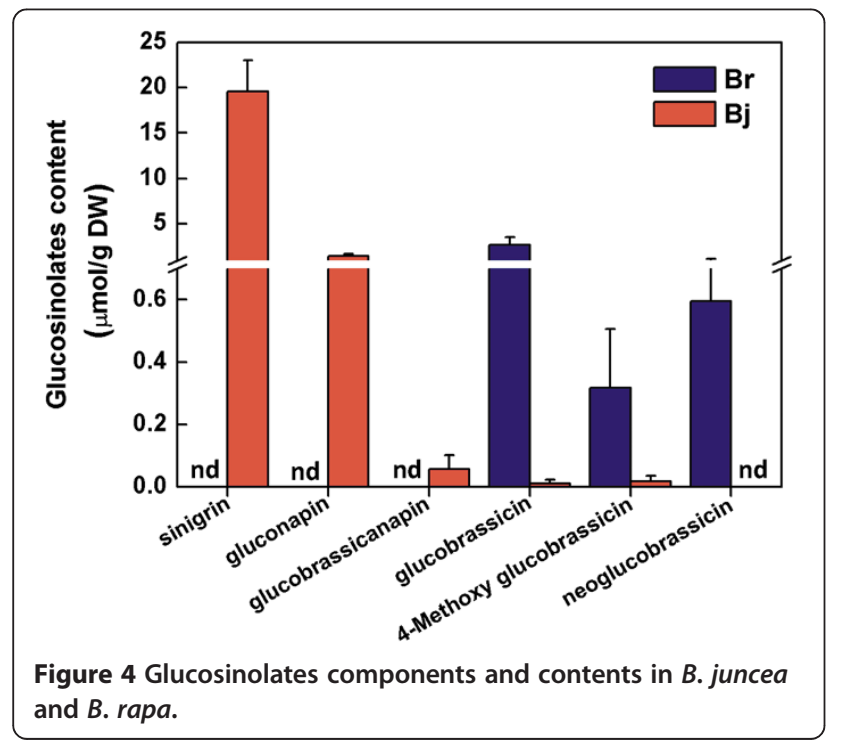

interest. Largely because of the vast phenotypic diversity available within the Brassicas [1,6].

The fate of duplicated genes can be defined as sub-functionalization, neo-functionalization or nonfunctionalized after polyploidization or whole genome duplication (WGD) in polyploid crops [36,37]. Biased gene expression between homologous gene is usually observed in allopolyploid plants, including Gossypium [38], Arabidopsis [39] and Tragopogon [40], resulting from genetic and epigenetic interactions between redundant genes, and these interactions can influence plant phenotypes and evolutionary fates of polyploid types [37]. Among the many models that attempt to explain how/ why duplicated genes are retained after polyploidy [41], sub-functionalization is the most popular hypothesis even though it remains controversial [42]. Genome plasticity, redundancy and diversity are well described and discussed in polyploid Brassicaceae [43-45], and are thought to contribute to adaptive phenotypic variation $[37,40,46]$. For example, flowering time variation is affected by the replicated copies of the flowering time gene FLC in Brassicaceae [46]. Here, we preliminarily show that vernalization-dependent and photoperioddependent flowering pathways coexist in allopolyploid $B$. juncea, suggesting that the flowering pathways of $B$. rapa and $B$. nigra can express in independent vernalization environments in the allopolyploid B. juncea. Timing of flowering onset is an essential trait that affects crop production and plant life cycle. To meet the challenges of climate changes and adapt to a wider range of growing environments, plants adjust their flowering time or pathway during evolution. The coexistence of vernalizationdependent and photoperiod-dependent flowering pathways 


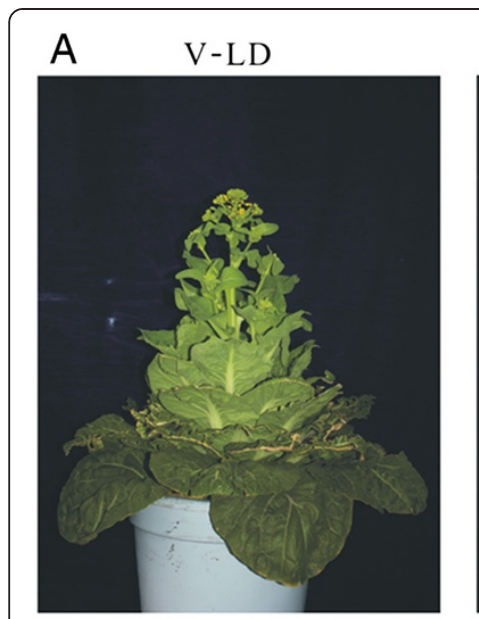

B. rapa

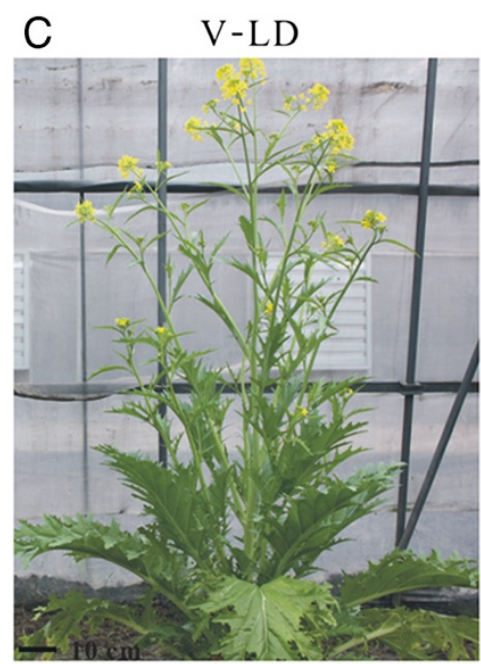

B. juncea

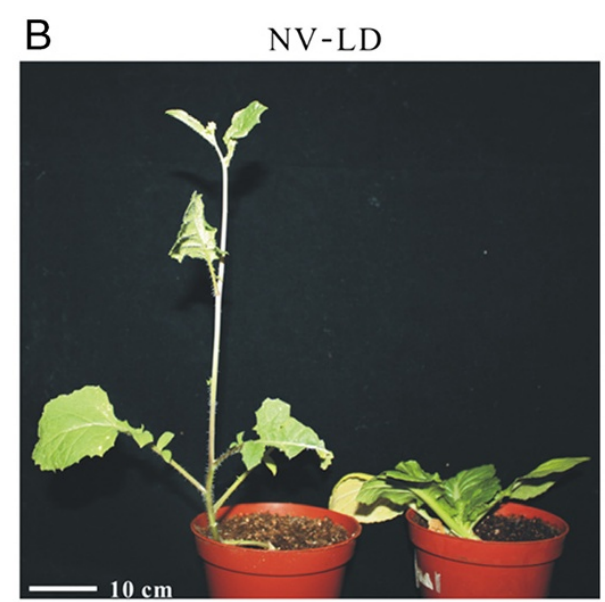

B. nigra
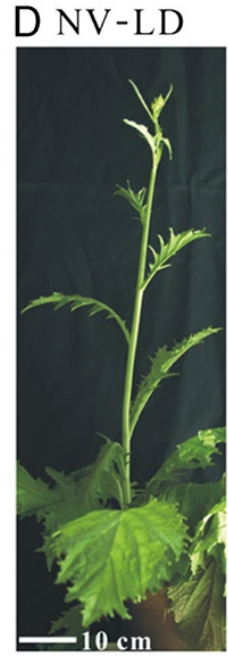

B. juncea

F
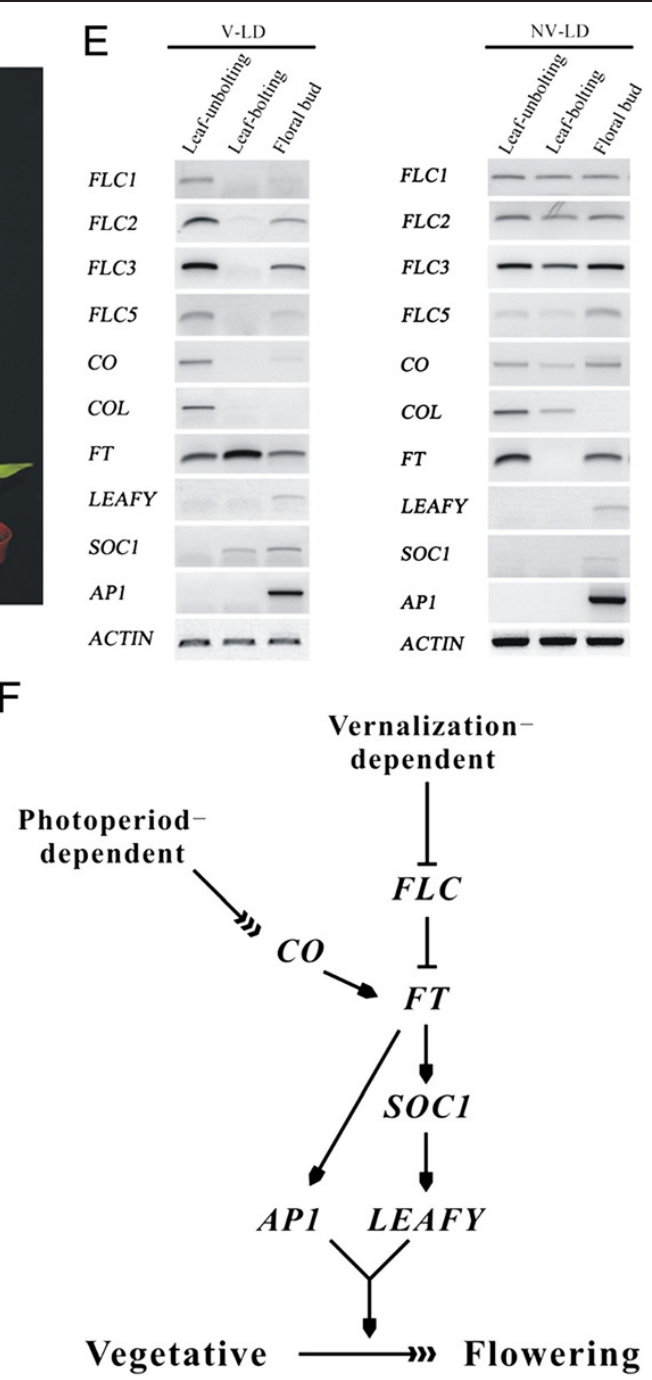

Figure $\mathbf{5}$ Vernalization- and photoperiod-dependent flowering pathways coexist in allotetraploid $\mathbf{B}$. juncea. Vernalization and long-day photoperiod condition (A and $\mathbf{C}$ ), Non-vernalization and long-day photoperiod condition (B and $\mathbf{D}), B$. juncea flowering pathway related gene expression (E) and proposed flowering pathway in B. juncea (F).

might indicate better adaptation for survival during evolution in B. juncea. On the other hand, with global warming, B. juncea may have more potential to be used as oil crops because of its flowering trait independent of vernalization status.

In this study, we employed high-throughput sequencing approach based on Illumina/Solexa platform to investigate $30 \times$ genome survey sequences of $B$. juncea. After comparison to $B$. rapa genome, $45.93 \%$ genome survey sequences of $B$. juncea can be mapped to B. rapa genome, which indicate relative far phylogenetic relationship between $\mathrm{A} / \mathrm{B}$ than $\mathrm{A} / \mathrm{C}$. This provides an opportunity that we can sequence this genome by diploid approaches. After comparative genome analysis between $B$. juncea and $B$. rapa, we find more SV in $B$. juncea genome, which may be resulted from polyploidy event. Moreover, based on the
$30 \times$ genome survey sequences of $B$. juncea, we observed huge polymorphisms between $B$. juncea and $B$. rapa including SNPs, SVs and Indels. The non-synonymous SNPs, frame-shift Indels and genes with SVs resulted from these polymorphisms caused a large number of pathways to be changed in B. juncea by KEGG analysis, for example, glucosinolate biosynthesis pathway. Higher expressions of CYP83A1 and CYP79F1 genes are associated with a higher content of aliphatic glucosinolate in B. juncea than B.rapa. Increased $C Y P 83 B 1$ gene expression is associated with a higher content of indole glucosinolate in Brapa than $B$. juncea. However, we did not observe a higher content of aromatic glucosinolate resulting from observed higher expression of CYP79A2 in B. juncea than B. rapa. That may be reason that we did not observe higher expressions of SUR1 downstream of CYP79A2 and CYP79B2 in aromatic 
glucosinolate biosynthesis pathway in $B$. juncea. The advent of high-throughput sequencing (Next-generation sequencing, NGS) has revolutionized genomic and transcriptomic approaches to biology. These new sequencing tools are also valuable for discovering, sequencing and genotyping not only hundreds but thousands of markers across almost any genome of interest, even in species in which little or no genetic information is available [47].

\section{Conclusions}

In this study, we find the clues of glucosinolate biosynthesis and flowering pathways evolution in B. juncea based on comparative analysis between $30 \times$ genome survey sequences of $B$. juncea and genome of B. rapa, which allow us to propose that polyploidization resulted in the evolution of glucosinolate biosynthesis and flowering pathways in $B$. juncea. The genome survey sequencings promote the whole genome sequencing processing in B. juncea. To conclude, next-generation sequencing, even low genome coverage is pushing forward the molecular genetics especially in non-model plant.

\section{Methods}

\section{Plant materials}

The inbred line of Brassica jucnea var tumida Tsen et Lee from our lab (Institute of Vegetable Science, Zhejiang University) was used to conduct genome survey sequencing in this study. Brassica rapa and Brassica nigra seeds were procured from the University of Warwich and Beijing Academy of Agriculture and Forestry Sciences, respectively.

\section{Genome in situ hybridization of chromosome in B. juncea}

Seeds of $B$. juncea, B. rapa and B. nigra were germinated at $28^{\circ} \mathrm{C}$ in dark. Root tips were harvested, in ice-bath for 24 hours and fixed in solution (Ethanol: Acetic acid =3: 1) for 24 hours. The root tips were stained within $1 \%$ acetocarmine for $15 \mathrm{~min}$ and dropped on slide with $45 \%$ acetic acid then covered with a coverslip. The slides with samples were examined by microscope to find the metaphase stage of chromosome and then conserved. Total genomic DNA was isolated from young leaf tissue of $B$. rapa and B. nigra using a DNA extraction kit (QIAGEN, USA). The genomic DNA of $B$. rapa was labeled with biotin-16-dUTP by nick translation and the genomic DNA of B. nigra was labeled with digoxingenin-11-dUTP by nick translation (Roche, USA). For genomic in situ hybridization, slide pretreatment, chromosome denaturation with probe, hybridization and post-hybridization treatments were referred to the method [48]. The images were captured and analyzed using Zeiss Axioskop fluorescence microscope system (ZEISS, Germany).

\section{Library construction, sequencing and re-sequencing}

Genomic DNAs were isolated from young leaf tissue of B. juncea using a DNA extraction kit (Illunima, USA).
Genomic Paired-end libraries with 170 bp and 500 bp insertion were constructed following a standard protocol provided by Illumina. The adapter ligation and DNA cluster preparation were performed and subjected to sequencing using Illumina Genome Analyzer (Illumina Hiseq2000, USA) according to the manufacturer's standard protocol. Low-quality reads, reads with adaptor sequences and duplicated reads were filtered, and remaining high-quality data was used in the following assembly and analysis.

\section{Comparative genome analysis}

Genome sequence of $B$. rapa was used as reference to comparatively analyze the genome survey sequences (GSS) of B. juncea by using Burrows-Wheeler Aligner (BWA) program. Samtools, Pindel and Breakdancer software were used to analyze the molecular polymorphisms including SNP, SV and Indel polymorphisms by comparison of the survey genome of $B$. juncea and genome of $B$. rapa. BLAST software was used for gene annotation.

\section{Glucosinolate biosynthesis gene expression}

Total RNA was extracted from seedlings using an RNeasy Plant Mini Kit (QIAGEN, USA) following the manufacturer's protocol. During extraction, total RNA was exhaustively treated with RNase-free Dnase (Qiagen, Germany). RNA concentration and quality were determined with a biophotometer (Eppendorf, Germany) and gel analysis. $1 \mu \mathrm{g}$ total RNA was transcribed to synthesize cDNA first strand using a Reverse Transcriptase M-MLV Kit (Takara, Japan). The expression of 6 selected genes was assayed in B. juncea and B. rapa by quantitative real-time PCR (qPCR) on ABI Step One (Applied Biosystems, USA). qPCR reaction were performed using $2.5 \mu \mathrm{l}$ cDNA template, $6.5 \mu \mathrm{l}$ of Fast start universal SYBR Green Master (Roche Germany), and $2.0 \mu \mathrm{M}$ primer, in a total $20 \mu \mathrm{l}$ reaction system. The relative quantification of the target gene was determined using the ${ }^{\Delta \Delta} \mathrm{CT}$ method. All PCR reactions were run in triplicate on each plate as technical replicates and three independent biological replicates were used. Gene fragment of CYP83A1, CYP79A2, CYP83B1, CYP79B2, CYP79F1, SUR1 and 25S were cloned from $B$. juncea and $B$. rapa and conserved sequences of these genes were used for primer design. 25S was used as an internal control gene to evaluate relative gene expression level. Primers used in this study are listed in Additional file 1: Table S6.

\section{Glucosinolate content measurement}

Duplicates of the freeze-dried powder $(0.25 \mathrm{~g})$ in $10 \mathrm{ml}$ glass tubes were preheated for $5 \mathrm{~min}$ in $75^{\circ} \mathrm{C}$ water bath. And $4 \mathrm{ml}$ of $70 \%$ boiling methanol $\left(75^{\circ} \mathrm{C}\right)$ were added and extracted at $75^{\circ} \mathrm{C}$ in a water bath for $10 \mathrm{~min}$. For internal standardization $100 \mu \mathrm{l}$ of $5 \mathrm{mM}$ sinigrin (Sigma-Aldrich 
Co., MO, USA) were added to one of the duplicates before extraction. Then $1 \mathrm{ml}$ of $0.4 \mathrm{M}$ barium acetate were rapidly added and the vials vortexed for several seconds. After centrifugation at $4,000 \mathrm{rpm}$ for $10 \mathrm{~min}$ at room temperature, the supernatants were collected and the pellets were re-extracted twice with $3 \mathrm{ml}$ of $70 \%$ boiling methanol $\left(75^{\circ} \mathrm{C}\right)$. Three supernatants were combined and made up to a final volume of $10 \mathrm{ml}$ with $70 \%$ methanol. $5 \mathrm{ml}$ extracts were loaded onto a $1 \mathrm{ml} \mathrm{mini-column} \mathrm{(JT}$ Baker, USA) containing $500 \mu \mathrm{l}$ of activated DEAE Sephadex $^{\text {tx }}$ A-25 (Amersham Biosciences, Sweden), and allowed to desulphate overnight with aryl sulfatase (Sigma-Aldrich Co., MO, USA). The resultant desulpho (ds)-GS were eluted with $2.5 \mathrm{ml}$ of ultra pure water produced by Milli-Q system (Millipore Co., USA) and stored at $-20^{\circ} \mathrm{C}$ prior to separation by high performance liquid chromatography (HPLC).

Samples of $40 \mu \mathrm{l}$ were analyzed in a Shimadzu HPLC system (LC-10AT pump, CTO-10A column oven, SCL10A VP system controller, Shimadzu, Kyoto, Japan) consisting of a UV-VIS detector (SPD-10A) set at $229 \mathrm{~nm}$ and a prontosil ODS2 column $(250 \times 4 \mu \mathrm{m}, 5 \mu \mathrm{m}$, Bischoff, Germany). The mobile phase consisted of ultrapure water (A) and acetonitrile (Tedia, USA) (B). The mobile phase was in the following gradient: $\mathrm{H}_{2} \mathrm{O}(2 \mathrm{~min})$, a linear gradient of $0-20 \%$ acetonitrile (32 $\mathrm{min}), 20 \%$ acetonitrile $(6 \mathrm{~min})$, followed by $100 \%$ acetonitrile and $0 \%$ acetonitrile prior to the injection of the next sample.

\section{Identification of flowering pathway in B. juncea}

For vernalization and long-day treatment, $B$. juncea and $B$. rapa were grown in glass greenhouse during winter season starting 4th week of October. Under these conditions, B. rapa began flowering in March and B. juncea in April. For non-vernalization and long-day treatment, $B$. juncea, $B$. nigra and $B$. rapa were grown in a growth chamber under conditions of $25^{\circ} \mathrm{C}$ and photoperiod of 16 light: 8 dark. Semi-RT-PCR method was employed to study the flowering pathway-related gene expression, including FLC1/2/3/5, CO, COL, FT, LEAFY, SOC1, AP1. ACTIN gene from B. juncea was used as an internal control gene to evaluate relative gene expression level. Degenerate primers of flowering pathway-related genes were referred to publications [49-51], NCBI Accessions JQ314107, JN699544 and cloned gene fragment. Primers of ACTIN gene was designed by NCBI Accessions HM565958. Primers used in this study are listed in Supporting Information Additional file 1: Table S6.

\section{Additional file}

Additional file 1: Table S1. Statistical comparison of sequencing reads of $B$. juncea with genome of B. rapa. Table S2: Statistic of SNPs between mapped sequences of $B$. juncea and B. rapa genome sequence. Table S3:
Statistic of SVs between mapped sequences of B. juncea and B. rapa genome sequence. Table S4: Statistic of Indel ( $1-3$ bp) between mapped sequences of $B$. juncea and B. rapa genome sequence. Table S5: Polymorphism information on glucosinolate biosynthesis related genes between $B$. juncea and B. rapa. Table S6: Primer sequences used for qRT-PCR and RT-PCR. Figure S1: Estimation of high-throughput sequencing quality including insert size, quality distribution, nucleotide content and cycle average quality distribution. Figure S2: Comparison of in-depth distribution of sequencing reads from $B$. juncea on chromosome of $B$. rapa. Figure S3: Distribution of SNP and SV polymorphisms on chromosome of B. rapa. Figure S4: Distribution of 1-3 bp Indels in genome and coding sequence (CDS) region of B. rapa.

\section{Competing interests}

The authors have declared that no competing interests exist.

\section{Authors' contributions}

$J Y$ and $M Z$ conceived and designed the experiments. JY, NS, XZ, XQ and ZH performed the experiments and data analysis. JY wrote the paper and MZ edited the paper. All authors read and approved the final manuscript.

\section{Acknowledgements}

We thank to Mr. H He from Biomarker Technologies Inc. for comparative genome analysis. We thank to Dr. YX Zang from Zhejiang A\&F University for measuring the contents of glucosinolates. We express gratitude to Prof. Dr. Y Mukai and G Suzuki from Osaka Kyoiku University for GISH analysis. We also thank to Prof. Sally A. Mackenzie from University of Nebraska-Lincoln for her critical comments and editing of this paper.

\section{Funding}

This work was supported by grants from Qianjiang Talents Project of Science Technology Department of Zhejiang Province (2012R10024), the National Natural Science Foundation of China (30971994).

\section{Author details}

'Laboratory of Germplasm Innovation and Molecular Breeding, Institute of Vegetable Science, Zhejiang University, Hangzhou 310058, P. R. China. ${ }^{2}$ Key laboratory of Horticultural Plant Growth, Development \& Quality

Improvement, Ministry of Agriculture, Hangzhou 310058, P. R. China. ${ }^{3}$ College of Horticulture and Plant Protection, Yangzhou University, Yangzhou 225009, P. R. China.

Received: 17 August 2013 Accepted: 23 January 2014

Published: 6 February 2014

\section{References}

1. Beilstein MA, Al-Shehbaz IA, Kellogg EA: Brassicaceae phylogeny and trichome evolution. Am J Bot 2006, 93(4):607-619.

2. Johnston JS, Pepper AE, Hall AE, Chen ZJ, Hodnett G, Drabek J, Lopez R, Price HJ: Evolution of genome size in Brassicaceae. Ann Bot-London 2005, 95(1):229-235.

3. Lysak MA, Koch MA, Beaulieu JM, Meister A, Leitch IJ: The Dynamic Ups and Downs of Genome Size Evolution in Brassicaceae. Mol Biol Evol 2009, 26(1):85-98.

4. Schranz ME, Lysak MA, Mitchell-Olds T: The ABC's of comparative genomics in the Brassicaceae: building blocks of crucifer genomes. Trends Plant Sci 2006, 11(11):535-542.

5. Wang $X$, Wang $H$, Wang J, Sun R, Wu J, Liu S, Bai Y, Mun JH, Bancroft I, Cheng $F$, et al: The genome of the mesopolyploid crop species Brassica rapa. Nat Genet 2011, 43(10):1035-1039.

6. Nagaharu U: Genome analysis in Brassica with special reference to the experimental formation of B. napus and peculiar mode of fertilization. Japan Journal of Botany 1935, 7:389-452.

7. O'Neill CM, Bancroft I: Comparative physical mapping of segments of the genome of Brassica oleracea var. alboglabra that are homoeologous to sequenced regions of chromosomes 4 and 5 of Arabidopsis thaliana. Plant J 2000, 23(2):233-243.

8. Town CD, Cheung F, Maiti R, Crabtree J, Haas BJ, Wortman JR, Hine EE, Althoff R, Arbogast TS, Tallon LJ, et al: Comparative genomics of Brassica 
oleracea and Arabidopsis thaliana reveal gene loss, fragmentation, and dispersal after polyploidy. Plant Cell 2006, 18(6):1348-1359.

9. Yang TJ, Kim JS, Kwon SJ, Lim KB, Choi BS, Kim JA, Jin M, Park JY, Lim MH, $\mathrm{Kim} \mathrm{HI}$, et al: Sequence-level analysis of the diploidization process in the triplicated FLOWERING LOCUS C region of Brassica rapa. Plant Cell 2006, 18(6):1339-1347.

10. Mun JH, Kwon SJ, Yang TJ, Seol YJ, Jin M, Kim JA, Lim MH, Kim JS, Baek S, Choi BS, et al: Genome-wide comparative analysis of the Brassica rapa gene space reveals genome shrinkage and differential loss of duplicated genes after whole genome triplication. Genome Biol 2009, 10(10):R111.

11. Qi XH, Zhang MF, Yang JH: Molecular phylogeny of Chinese vegetable mustard (Brassica juncea) based on the internal transcribed spacers (ITS) of nuclear ribosomal DNA. Genet Resour Crop Ev 2007, 54(8):1709-1716.

12. Antonious GF, Bomford M, Vincelli P: Screening Brassica species for glucosinolate content. J Environ Sci Health B 2009, 44(3):311-316.

13. Verkerk R, Schreiner M, Krumbein A, Ciska E, Holst B, Rowland I, De Schrijver R, Hansen M, Gerhauser C, Mithen R, et al: Glucosinolates in Brassica vegetables: the influence of the food supply chain on intake, bioavailability and human health. Mol Nutr Food Res 2009, 53:S219-S265.

14. Bending GD, Lincoln SD: Characterization of volatile sulphur-containing compounds produced during decompositioin of Brassica juncea tissues in soil. Soil Biology \& Biochemistry 1999, 31:695-703.

15. Kaul S, Koo HL, Jenkins J, Rizzo M, Rooney T, Tallon LJ, Feldblyum T, Nierman W, Benito MI, Lin XY, et al: Analysis of the genome sequence of the flowering plant Arabidopsis thaliana. Nature 2000, 408(6814):796-815

16. Parkin IAP, Gulden SM, Sharpe AG, Lukens L, Trick M, Osborn TC, Lydiate DJ: Segmental structure of the Brassica napus genome based on comparative analysis with Arabidopsis thaliana. Genetics 2005, 171(2):765-781.

17. Lagercrantz U, Lydiate DJ: Comparative genome mapping in Brassica. Genetics 1996, 144(4):1903-1910.

18. Lagercrantz U: Comparative mapping between Arabidopsis thaliana and Brassica nigra indicates that Brassica genomes have evolved through extensive genome replication accompanied by chromosome fusions and frequent rearrangements. Genetics 1998, 150(3):1217-1228.

19. Lan TH, DelMonte TA, Reischmann KP, Hyman J, Kowalski SP, McFerson J, Kresovich S, Paterson AH: An EST-enriched comparative map of Brassica oleracea and Arabidopsis thaliana. Genome Research 2000, 10(6):776-788

20. Babula D, Kaczmarek M, Barakat A, Delseny M, Quiros CF, Sadowski J: Chromosomal mapping of Brassica oleracea based on ESTs from Arabidopsis thaliana: complexity of the comparative map. Mol Genet Genomics 2003, 268(5):656-665.

21. Panjabi P, Jagannath A, Bisht NC, Padmaja KL, Sharma S, Gupta V, Pradhan AK, Pental D: Comparative mapping of Brassica juncea and Arabidopsis thaliana using Intron Polymorphism (IP) markers: homoeologous relationships, diversification and evolution of the $A, B$ and $C$ Brassica genomes. BMC Genomics 2008, 9:113.

22. Lukens L, Zou F, Lydiate D, Parkin I, Osborn T: Comparison of a Brassica oleracea genetic map with the genome of Arabidopsis thaliana. Genetics 2003, 164(1):359-372.

23. Boivin K, Acarkan A, Mbulu RS, Clarenz O, Schmidt R: The Arabidopsis genome sequence as a tool for genome analysis in Brassicaceae. A comparison of the Arabidopsis and Capsella rubella genomes. Plant Physiol 2004, 135(2):735-744

24. Lysak MA, Berr A, Pecinka A, Schmidt R, McBreen K, Schubert I: Mechanisms of chromosome number reduction in Arabidopsis thaliana and related Brassicaceae species. P Natl Acad Sci USA 2006, 103(13):5224-5229.

25. Lynch $M$, Force $A$ : The probability of duplicate gene preservation by subfunctionalization. Genetics 2000, 154(1):459-473.

26. He XL, Zhang JZ: Rapid subfunctionalization accompanied by prolonged and substantial neofunctionalization in duplicate gene evolution. Genetics 2005, 169(2):1157-1164.

27. Milinkovitch MC, Helaers R, Depiereux E, Tzika AC, Gabaldon T: 2x genomes-depth does matter. Genome Biol 2010, 11(2):R16.

28. Cheng X, Xu J, Xia S, Gu J, Yang Y, Fu J, Qian X, Zhang S, Wu J, Liu K: Development and genetic mapping of microsatellite markers from genome survey sequences in Brassica napus. Theor Appl Genet 2009, 118(6):1121-1131.

29. Kirkness EF, Bafna V, Halpern AL, Levy S, Remington K, Rusch DB, Delcher AL, Pop M, Wang W, Fraser CM, et al: The dog genome: survey sequencing and comparative analysis. Science 2003, 301(5641):1898-1903.
30. Venkatesh B, Kirkness EF, Loh YH, Halpern AL, Lee AP, Johnson J, Dandona $\mathrm{N}$, Viswanathan LD, Tay A, Venter JC, et al: Survey sequencing and comparative analysis of the elephant shark (Callorhinchus milii) genome. PLOS Biol 2007, 5(4):e101.

31. Wicker T, Narechania A, Sabot F, Stein J, Vu GT, Graner A, Ware D, Stein N: Low-pass shotgun sequencing of the barley genome facilitates rapid identification of genes, conserved non-coding sequences and novel repeats. BMC Genomics 2008, 9:518.

32. Bouck J, Miller W, Gorrell JH, Muzny D, Gibbs RA: Analysis of the quality and utility of random shotgun sequencing at low redundancies. Genome Res 1998, 8(10):1074-1084.

33. Wernersson R, Schierup MH, Jorgensen FG, Gorodkin J, Panitz F, Staerfeldt $\mathrm{HH}$, Christensen OF, Mailund T, Hornshoj $\mathrm{H}$, Klein A: Pigs in sequence space: a $0.66 \mathrm{X}$ coverage pig genome survey based on shotgun sequencing. BMC Genomics 2005, 6:70.

34. Rasmussen DA, Noor MAF: What can you do with $0.1 \times$ genome coverage? A case study based on a genome survey of the scuttle fly Megaselia scalaris (Phoridae). BMC Genomics 2009, 10:382.

35. Yu JY, Zhao MX, Wang XW, Tong CB, Huang SM, Tehrim S, Liu YM, Hua W, Liu SY: Bolbase: a comprehensive genomics database for Brassica oleracea. BMC Genomics 2013, 14:664

36. Jackson S, Chen ZJ: Genomic and expression plasticity of polyploidy. Curr Opin Plant Biol 2010, 13(2):153-159.

37. Comai $L$ : The advantages and disadvantages of being polyploid. Nature Reviews Genetics 2005, 6(11):836-846.

38. Chaudhary B, Flagel L, Stupar RM, Udall JA, Verma N, Springer NM, Wendel $J F$ : Reciprocal silencing, transcriptional bias and functional divergence of homeologs in polyploid cotton (gossypium). Genetics 2009, 182(2):503-517.

39. Blanc $\mathrm{G}$, Wolfe $\mathrm{KH}$ : Functional divergence of duplicated genes formed by polyploidy during Arabidopsis evolution. Plant Cell 2004, 16(7):1679-1691.

40. Buggs RJA, Elliott NM, Zhang LJ, Koh J, Viccini LF, Soltis DE, Soltis PS: Tissue-specific silencing of homoeologs in natural populations of the recent allopolyploid Tragopogon mirus. New Phytol 2010, 186(1):175-183.

41. Freeling $\mathrm{M}$ : Bias in plant gene content following different sorts of duplication: tandem, whole-genome, segmental, or by transposition. Annu Rev Plant Biol 2009, 60:433-453.

42. Freeling M: The evolutionary position of subfunctionalization, downgraded. Genome Dyn 2008, 4:25-40.

43. Lukens LN, Quijada PA, Udall J, Pires JC, Schranz ME, Osborn TC: Genome redundancy and plasticity within ancient and recent Brassica crop species. Biol J Linn Soc 2004, 82(4):665-674.

44. Leitch AR, Leitch IJ: Genome plasticity and the diversity of polyploid plants. Science 2008, 320:481-483

45. Marhold K, Lihová J: Polyploidy, hybrization and reticulated evolution: lessons from the Brassicaceae. Plant Systematics and Evolution 2006, 259:143-174.

46. Osborn TC: The contribution of polyploidy to variation in Brassica species. Physiologia Plantatum 2004, 121:531-536.

47. Davey JW, Hohenlohe PA, Etter PD, Boone JQ, Catchen JM, Blaxter ML: Genome-wide genetic marker discovery and genotyping using nextgeneration sequencing. Nat Rev Genet 2011, 12(7):499-510.

48. Howell EC, Kearsey MJ, Jones GH, King GJ, Armstrong SJ: A and C genome distinction and chromosome identification in brassica napus by sequential fluorescence in situ hybridization and genomic in situ hybridization. Genetics 2008, 180(4):1849-1857.

49. Martynov W, Khavkin EE: Polymorphism of the CONSTANS gene in Brassica plants. Russ J Plant Phys/+ 2005, 52(2):242-248.

50. Vorobiev VA, Martynov W, Pankin AA, Khavkin EE: Polymorphism of the LEAFY gene in Brassica plants. Russ J Plant Phys/+ 2005, 52(6):814-820.

51. Schranz ME, Quijada P, Sung SB, Lukens L, Amasino R, Osborn TC: Characterization and effects of the replicated flowering time gene FLC in Brassica rapa. Genetics 2002, 162(3):1457-1468.

doi:10.1186/1471-2164-15-107

Cite this article as: Yang et al:: Genome survey sequencing provides clues into glucosinolate biosynthesis and flowering pathway evolution in allotetrapolyploid Brassica juncea. BMC Genomics 2014 15:107. 